\title{
Reduced Outpatient Obstetrics and Gynecology Visits During COVID-19 Pandemic in a Medium-Sized Regional Hospital, Japan
}

\author{
Satoshi Ichigo ${ }^{1}$, Ichiro Kawabata ${ }^{1}$, Kazutoshi Matsunami ${ }^{1}$, Hiroshi Takagi ${ }^{1}$, Hazuki Kagawa ${ }^{2}$, \\ Yuji Ito ${ }^{2}$ and Atsushi Imai ${ }^{1 *}$ \\ ${ }^{1}$ Department of Obstetrics and Gynecology, Matsunami General Hospital, Japan
}

${ }^{2}$ Department of Obstetrics and Gynecology, Ena Municipal Hospital, Japan

*Corresponding author: Atsushi Imai, Department of Obstetrics and Gynecology, Matsunami General Hospital, 185-1 Dendai,

Kasamatsu, Gifu 501-6062, Japan

\section{ARTICLE INFO}

Received: 慧 October 25, 2021

Published: 幽 November 08, 2021

Citation: Satoshi Ichigo, Ichiro Kawabata, Kazutoshi Matsunami, Hiroshi Takagi, Atsushi Imai, et al., Reduced Outpatient Obstetrics and Gynecology Visits During COVID-19 Pandemic in a MediumSized Regional Hospital, Japan. Biomed J Sci \& Tech Res 39(5)-2021. BJSTR. MS.ID.006360.

\section{ABSTRACT}

Background: The COVID-19 pandemic has caused substantial disruption to many healthcare services. The purpose of this brief report was to describe changes in the number of outpatient obstetrics and gynecology visits at the medium-sized regional hospital during the COVID-19 pandemic.

Methods: Outpatient visit data was retrieved from registries of the medical center and the obstetrics and gynecology department. We compared the number of visits from April 2020 to September 2021 (pandemic period) with those from January 2018 to March 2020 (pre-pandemic).

Results: During the pre-pandemic period, approximately $10 \%$ of total visits received obstetrics and gynecology care. The number of visits during the initial 3 months of pandemic period was significantly lower than the pre-pandemic period (852 \pm 49.1 vs $1136 \pm 55.3, p<0.05)$. The refrain from consultation for obstetrics and gynecology care remained throughout the pandemic $(957 \pm 77.7)$.

Conclusion: The reductions in outpatient visits during the initial 3 months of COVID-19 pandemic appeared to reflect the patient's intention avoiding outpatient visits due to the anxiety of infection.

\section{Introduction}

The coronavirus pandemic crippled the healthcare delivery around the world [1,2]. Many countries, including Japan, implemented guidelines to diminish exposure, and individuals were encouraged to stay-at-home orders and self-quarantine to reduce transmission risk of infection, particularly those considered high risk for coronavirus infection (e.g. the elderly and immunocompromised). It brought about unprecedent transformed healthcare services, any form of routine medical care should be avoided and only emergency services may be provided [3,4]. Every country, region and state improved the guidelines according to their infection status and regional requirements [5]. Therefore, we attempted to critically investigate the changes in the number of the outpatient obstetrics and gynecology visits during the pandemic period compared to that of pre-pandemic period at the mid-sized care hospital in the region of Japan.

\section{Materials and Methods}

This prospective study was carried out by retrieving outpatient visit data from registries of Matsunami General Medical Center (Gifu, Japan) and the Department of Obstetrics and Gynecology. The data collected from January 2018 to March 2020 was categorized 
into the pre-pandemic period and the data from April 2020 to September 2021 was grouped into the pandemic period. The statistical analysis was performed with Student's t-test, and a P-value of $<0.05$ was considered significant.

\section{Results}

Total number of outpatient visits during the initial 3 months of COVID-19 pandemic decreased by17.3\% (from $20643 \pm 871.1$ to $17088 \pm 886.0$ per month, $\mathrm{p}<0.05$ ) compared to before COVID-19 pandemic (January 2018 to March 2020) (Figure1). There was no significant gender difference in pre-pandemic and pandemic periods. During the pre-pandemic period, approximately $10 \%$ of total visits received obstetrics and gynecologic care (Figure 2). The number during the initial 3 months of COVID-19 pandemic was significantly lower than the pre-pandemic period $(852 \pm 49.1$ vs $1136 \pm 55.3, \mathrm{p}<0.05)$. The refrain from consultation for obstetrics and gynecologic consultations had a slight tendency to remain throughout pandemic period (means of $957 \pm 77.7$ ).

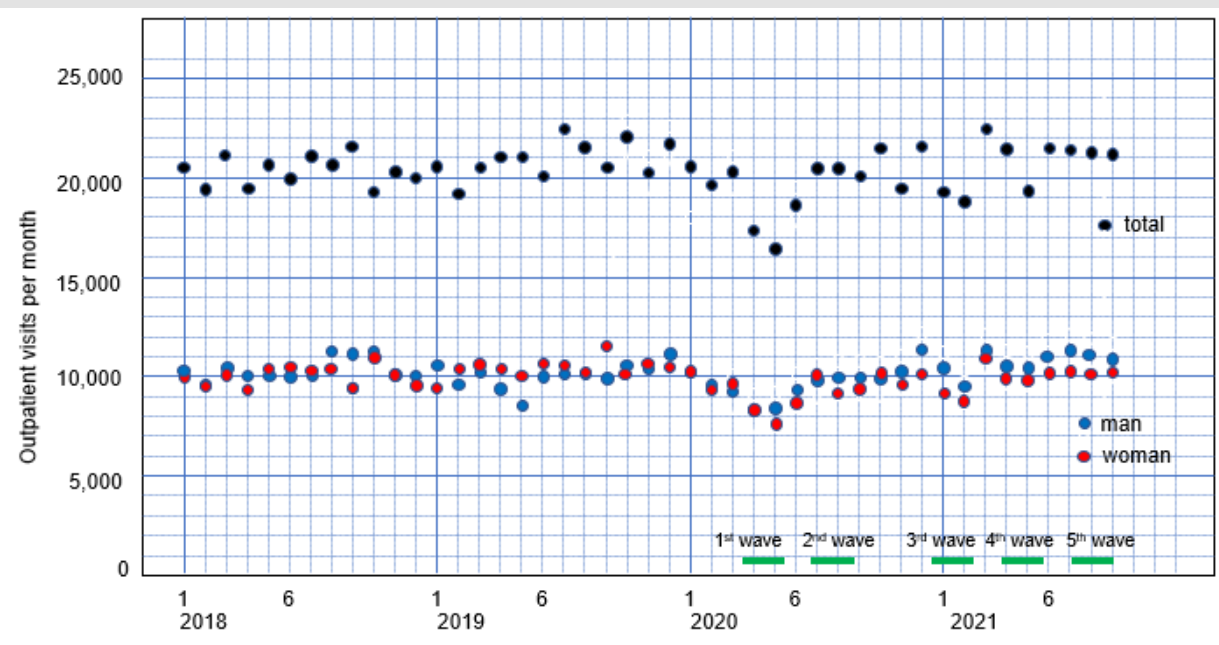

Figure 1: Medical center outpatient visits.

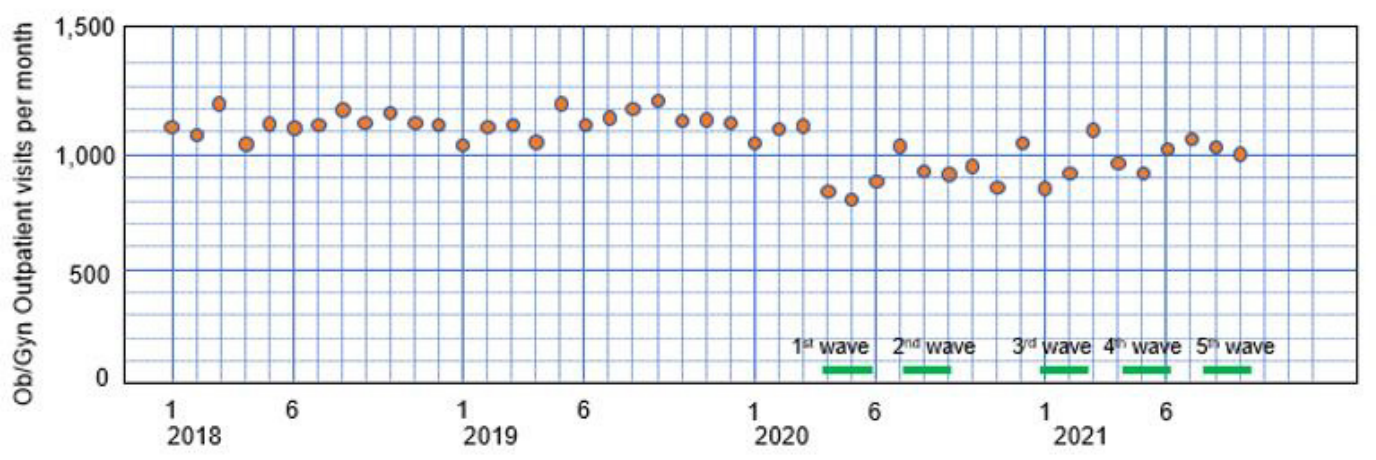

Figure 2: Obstetrics and gynecology outpatient visit.

\section{Discussion}

This retrospective study was designed to investigate the effect of the pandemic on the changing trends of obstetrics and gynecology outpatient. The analysis of patients in the pre-pandemic would reflect the usual pattern of cases attending our regional center and the drops in outpatient visits during the initial 3 months of COVID-19 pandemic seemed to more premiant than that of total institutional visits ( $17.3 \%$ vs $25.0 \%)$. This was followed by gradual relaxations that led to resumption of healthcare visits even under pandemic.
Restriction of movement during the initial 3 months of pandemic period prevented the patients from seeking prompt obstetrics and gynecology-related care and inability to receive the required treatment. Similar changing trend were also noticed in emergency or cancer cares [6-9]. In addition, patient may also intentionally avoid visiting hospitals due to scary infections. Awareness about the restriction of obstetrics and gynecology services during the initial pandemic period may lead to resumption of healthcare visits even under pandemic. Limitations of this study include that we did not categorize emergency, urgent and elective and that we did not 
observe the attendance age and clinical diagnosis. Further research is needed to understand how decreased outpatient visits during the pandemic may affect attendance outcomes.

\section{Conflict of Interest}

The authors declare that they have no conflict of interest.

\section{References}

1. Baloch S, Baloch MA, Zheng T, Pei X (2020) The coronavirus disease 2019 (COVID-19) pandemic. Tohoku J Exp Med 250(4): 271-278.

2. Wu D, Wu T, Liu Q Yang Z (2020) The SARS-Cov-2 outbreal: what we know. Int J Infect Dis 94: 44-48.

3. Hasöksüz M, Kiliç S, Saraç F (2020) Coronaviruses and SARS-COV-2. Turk J Med Sci 21 50(SI-1): 549-556.

ISSN: 2574-1241

DOI: $10.26717 /$ BJSTR.2021.39.006360

Atsushi Imai. Biomed J Sci \& Tech Res

(c) (P) This work is licensed under Creative

Submission Link: https://biomedres.us/submit-manuscript.php
4. Ali I, Alharbi OML (2020) COVID-19 disease. management, treatment and social impact. Sci Total Environ 728: 138861.

5. Chams N, Chams S, Badran R, Shams A, Araji A, et al. (2020) COVID-19: a multidisciplinary review. Front Public Health 8: 383.

6. Walker DM, Tolentino VR (2020) COVID-19: the impact on pediatric emergency care. Pediatr Emerg Med Pract 17(Suppl 6-1): 1-27.

7. Fusi Schmidhauser T, Preston NJ, Keller N, Gamondi C (2020) Conservative management of COVID-19 patient: emergency palliative care in action. J Pain Symptom Manage 60(1): e27-e30.

8. Patt D, Gordan L, Diaz M, Okon T, Grady L, et al. (2020) Impact of COVID-19 on Cancer Care: How the Pandemic Is Delaying Cancer Diagnosis and Treatment for American Seniors. JCO Clin Cancer Inform 4: 1059-1071.

9. Liu C, Zhao Y, Okwan Duodu D, Basho R, Cui X (2020) COVID-19 in cancer patients: risk, clinical features, and management. Cancer Biol Med 17(3): 519-527.

$\begin{array}{ll}\text { BIOMEDICAL } & \text { Assets of Publishing with us } \\ \text { RESEARCHES } & \text { - Global archiving of articles } \\ \text { - Immediate, unrestricted online access }\end{array}$

